\title{
Difficultés de la transition du lycée à l'université: Cas de l'enseignement des mathématiques au Maroc
}

\section{Difficulties of Transition from secondary school to university: Case of mathematics teaching in Morocco}

\author{
Brahim Nachit ${ }^{1,2, *}$, Jamal Harraq ${ }^{2,3}$ and Khalid hattaf ${ }^{2,3}$ \\ ${ }^{1}$ Laboratoire Interdisciplinaire de Recherche en Sciences, Education et Formation, Equipe \\ de recherche en Didactique des sciences, Ecole Supérieure d'Education et de Formation, \\ Université Hassan 1, Berrechid, Maroc. \\ ${ }^{2}$ Laboratoire d'Analyse, Modélisation et Simulation (LAMS), Faculté des Sciences de \\ Ben M'sik, Université Hassan II, Casablanca, Maroc. \\ ${ }^{3}$ Centre Régional des Métiers d'Education et de Formation, Casablanca-Settat, Maroc.
}

Résumé. La transition lycée - université est un moment difficile pour les étudiants poursuivant des études scientifiques ; particulièrement en mathématiques, c'est un problème délicat, reconnu à l'échelle internationale. La question de l'amélioration de la transition entre l'enseignement au lycée et l'enseignement à l'université concernant l'enseignement des mathématiques, ne date pas d'aujourd'hui, elle a été abordée par plusieurs mathématiciens et didacticiens. Le but de cette recherche est d'identifier et d'interpréter les difficultés des étudiants en mathématiques à la transition lycée - université. L'analyse des travaux dirigés et des cours de la première année scientifique, l'analyse des manuels de mathématiques de la dernière année du lycée et l'entretien semi-directif avec des professeurs de l'université, ont permis d'identifier les difficultés des étudiants de la première année universitaire et de déterminer les continuités et les ruptures dans les pratiques et modes d'enseignement de mathématiques, entre la dernière année du lycée et la première année des études supérieures et en particulier celle de l'université marocaine. Des recommandations ont été proposées pour prendre en charge certaines difficultés repérées lors de l'enseignement-apprentissage des mathématiques en première année universitaire, aider les élèves à préparer leur passage vers l'enseignement supérieur et diminuer le taux d'échec.

\begin{abstract}
The transition from high school to university is a difficult time for students pursuing scientific studies; particularly in mathematics. This is a problem, which is recognized internationally. The issue of improving the transition from high school to university education in the teaching of
\end{abstract}

* Corresponding author: nachitbrahim@yahoo.fr 
mathematics is not new and has been addressed by several mathematicians and didacticians. The purpose of this work is to identify and interpret the difficulties that mathematics students face during this transition from high school to university. In this regard, the analysis of tutorials and courses in the first scientific year, the analysis of the mathematics textbooks of the last year of the high school and the semi-structured interview with university professors, helped identify the difficulties of the university freshmen and determine the continuities and the ruptures in the practices and modes of teachings of mathematics between the last year of the high school and the first year of the higher studies and in particular that of the Moroccan university. Recommendations have been proposed to deal with certain difficulties identified in the teaching-learning of mathematics in the first year of university, to help students prepare for their transition to higher education and to reduce the failure rate.

\section{INTRODUCTION}

La transition lycée - université est un moment difficile pour les étudiants poursuivant des études scientifiques, particulièrement en mathématiques, c'est un problème reconnu à l'échelle internationale. Malgré l'importance d'une telle transition, nous enregistrons l'insuffisance des rencontres et des échanges entre les enseignants des deux niveaux d'études.

Depuis l'installation de la nouvelle réforme en 2003, le département de Mathématiques et Informatique a organisé des journées pédagogiques, en parallèle à des journées scientifiques, en présence de professeurs universitaires de Mathématiques de tout le Maroc et d'enseignants du secondaire, à la fin de toutes les années universitaires, pour évaluer l'enseignement des mathématiques à l'Université Marocaine.

Depuis juillet 2005, le Laboratoire d'Algèbre, Analyse et Applications L3A, le Laboratoire d'Analyse, Modélisation et Simulation (LAMS), le Laboratoire de Technologie de l'Information et Modélisation(LTIM) et l'Observatoire de Recherche en Didactique et Pédagogie Universitaire (ORDIPU) organisent chaque année une rencontre entre enseignants de Mathématiques du secondaire et du supérieur qui fait partie de " la transition secondaire/supérieur ».

Avec la création de l'association «Ben M'sik pour la promotion de la culture Mathématique et Informatique » (ABC-MATHINFO), en juillet 2010, il y avait organisation, par ses membres, de plusieurs journées concernant l'amélioration de l'enseignement supérieur, en particulier celui des mathématiques. L'objectif essentiel de ces journées, est de minimiser les impacts négatifs lors de la transition entre l'enseignement secondaire et celui de l'enseignement supérieur.

Des professeurs universitaires, enseignants et inspecteurs de l'enseignement secondaire et chercheurs en didactique des mathématiques participent à ces journées et rencontres pour débattre les thèmes de la transition. Elles ont toutes pour objectif d'identifier les différentes difficultés des étudiants de la première année universitaire en mathématiques et leurs causes et de proposer des moyens pour mieux réussir la transition secondaire/supérieur.

La question de la transition secondaire / supérieur n'est pas neuve, la didactique des mathématiques étudie depuis des années cette transition [1,2,3,4]. Cette étude s'inscrit dans le cadre d'une recherche menée sur la transition secondaire-supérieur au Maroc. Notre objectif est de décrire les facteurs qui influencent l'échec en mathématiques au début de l'enseignement à l'université. Ceci nous amène à poser les questions suivantes :

- Quelles sont les ruptures dans les pratiques et mode d'enseignement des mathématiques entre le lycée et la première année universitaire? 
- Quelles sont les difficultés que rencontrent la plupart des étudiants de la première année?

- Quelles sont les causes de ces difficultés?

- Comment réussir la transition lycée - université ?

\section{Cadre théorique}

Robert [5] a pointé l'arrivée massive à l'université d'un nouveau type de notions mathématiques : Formalisatrices, Unificatrices et Génératrices (FUG). Ces notions permettent en effet d'introduire plus de généralités, en unifiant différents objets antérieurs grâce à un nouveau formalisme. Ce formalisme permet de simplifier les écritures au détriment du sens.

Les tâches proposées aux élèves sont l'application immédiate des connaissances explicitées, tandis que l'étudiant doit les mélanger avec d'autres connaissances, introduire des intermédiaires, des étapes. La large taxonomie des tâches proposées aux étudiants rend la routinisation plus difficile. Selon Denise Grenier [6], il est nécessaire de soumettre les étudiants à des « situations recherches » qui font appel à l'expérimentation, la modélisation, la structuration, la formulation de conjectures, l'induction et la démonstration.

Bloch [7] a clarifié la complexité en terme de tâche par l'introduction des variables macro-didactiques pour mesurer la rupture entre le lycée et l'université.

Table 1. Variables macro-didactiques pour mesurer la rupture entre lycée et université.

\begin{tabular}{|c|c|c|}
\hline Variable didactique & Enseignement secondaire & Début de l'Université \\
\hline 0 . Introduction de la limite & Métaphores & Définition \\
\hline 1. Degré de formalisation & Faible & Elevé \\
\hline 2. Registre de validation & Algèbre des limites & Analyse \\
\hline 3. Degré de généralisation & Aucun & Elevé \\
\hline $\begin{array}{l}\text { 4. Introduction de nouveaux } \\
\text { savoirs }\end{array}$ & $\begin{array}{lcr}\text { Importante } & \text { (mais sans outils } \\
\text { théoriques } & \text { spécifiques } & \text { de } \\
\text { validation) } & & \\
\end{array}$ & $\begin{array}{l}\text { Importante (avec des outils } \\
\text { théoriques spécifiques de } \\
\text { validation) }\end{array}$ \\
\hline 5. Type de tâches & $\begin{array}{l}\text { Algorithme, tracé de graphiques, } \\
\text { calcul }\end{array}$ & Recherche et démonstration \\
\hline 6. Choix des techniques & Transparent & Amalgame \\
\hline $\begin{array}{l}\text { 7. Degré d'autonomie } \\
\text { nécessaire (ou sollicité) }\end{array}$ & $\begin{array}{llrr}\begin{array}{l}\text { Routines } \\
\text { techniques }\end{array} & \text { des } & \text { niveaux } \\
\text { mobilisables } & & & \text { au } \\
\end{array}$ & $\begin{array}{l}\text { Peu de routine à des niveaux } \\
\text { plutôt disponibles }\end{array}$ \\
\hline $\begin{array}{l}\text { 8. Mode d'intervention de la } \\
\text { notion }\end{array}$ & Processus & Objet \\
\hline $\begin{array}{l}\text { 9. Type de conversions entre } \\
\text { registres }\end{array}$ & Algébrique /Graphique & Algébrique/Analytique \\
\hline $\begin{array}{l}10 \text { Statut des énoncés } \\
\text { d'exercices }\end{array}$ & Application, instanciation & $\begin{array}{l}\text { Théorème, corollaire, énoncé } \\
\text { général }\end{array}$ \\
\hline
\end{tabular}

Bloch [7] ajoute que le passage de l'enseignement secondaire à l'enseignement supérieur s'accompagne de modifications majeures et que les étudiants sont confrontés à une révolution globale.

De point de vue macroscopique, on peut citer les ruptures suivantes : 
- Passage d'un cours avec un seul enseignant à des cours magistraux en amphi théâtre et des travaux dirigés ;

- Modularisation des enseignements qui contribue à isoler les connaissances les unes des autres et éventuellement plusieurs enseignants ;

- L'étudiant doit être plus autonome (travail personnel);

- Niveau de conceptualisation des connaissances à l'université est très élevé avec augmentation des exigences en terme de raisonnement, preuves et formalisation [8];

- Temps didactique accéléré : un renouvellement plus rapide des objets d'enseignement qui oblige une assimilation plus rapide [9] ;

- Manque d'encadrement des nouveaux étudiants ;

- La massification de l'enseignement à l'université ne facilite pas l'enseignement, l'apprentissage et le contact avec les enseignants ;

- Nouvel équilibre entre exercices à portée générale et exercices plus particuliers.

\section{Méthodologie}

L'étude a été réalisée à la Faculté des sciences Ben M'sick, et s'est basée sur :

- L'analyse des TD et cours de la première année scientifique ;

- L'analyse du programme et des manuels officiels du lycée option sciences expérimentale et sciences mathématiques ;

- L'analyse des orientations pédagogiques ;

- L'entretien semi-directif avec 40 professeurs de mathématiques à l'université. Cette technique nous a permis de rassembler les opinions des interviewés sur les difficultés des étudiants de la première année, les causes de ces difficultés et les solutions possibles pour réussir la transition lycée/université. Chaque entretien a duré environ $20 \mathrm{~min}$.

\section{Résultats et discussion}

Les difficultés auxquelles sont confrontés les étudiants de la première année universitaire sont souvent analysées comme une conséquence d'une préparation insuffisante des étudiants aux études supérieures.

Les entretiens avec les professeurs de mathématiques à l'université ont mis en évidence les principales causes de l'échec des nouveaux étudiants ;

- Hétérogénéité du niveau scientifique des diplômés du baccalauréat ;

- Mauvaise connaissance par les étudiants de la nature des études dans l'enseignement supérieur et sur le mode d'évaluation;

- Difficultés matérielles de certains étudiants (accès au logement, éloignement du foyer familial et difficultés de financement des études ;

- L'orientation au lycée est actuellement assurée par des conseillers d'orientation et des enseignants qui n'ont ni la formation, ni les outils du travail leur permettant d'orienter efficacement les élèves.

Robert [5] a défini trois niveaux de mise en fonctionnement des notions

- Le niveau technique : correspond à l'application immédiate de théorème, propriétés, définitions et formules ;

- Le niveau mobilisable : correspond à des mises en fonctionnement de plusieurs propriétés à la fois ;

- Le niveau disponible : exige que l'apprenant soit capable de résoudre ce qui lui est proposé sans aucune indication. Le niveau disponible est rarement présent au secondaire. 
$\mathrm{Au}$ lycée, les connaissances ne sont pas disponibles, l'apprentissage repose sur la reproduction des techniques et des exercices proches des examens.

L'analyse du programme et des manuels du lycée, des cours et des travaux dirigés de mathématiques de la première année universitaire nous a permis d'identifier les difficultés des étudiants de la première année en algèbre, en analyse, en logique et en géométrie et les ruptures dans les pratiques et modes d'enseignements des mathématiques entre le lycée et la première année universitaire.

Les manuels du lycée proposent en général des exercices usant les mêmes techniques dans des contextes très ciblés, les exercices proposés à la première année mettent en lumière un amalgame de techniques plus complexes, ils font appel à une forte formalisation, à un raisonnement par l'absurde et à la recherche de contre-exemples.

\subsection{En Analyse}

En analyse, les étudiants sont confrontés à de nouveaux objets et à de nouveaux modes de raisonnement [10], l'analyse des manuels de la dernière année du lycée montre que l'étude des fonctions est basée sur les calculs algébriques (calcul des limites, dérivés ...). Coppe et al [11] affirment que la forte algébrisation des techniques pour l'étude des fonctions est fort présente au niveau du Baccalauréat. L'étude est limitée à des objets isolés, le système de validation est basé sur l'algèbre des limites, tandis qu'à l'université, il est basé sur les définitions formelles et les approximations.

Les connaissances du monde fonctionnel restent très restreintes, les nouveaux étudiants ont l'habitude de réaliser des tâches bien précises, de travailler sur des fonctions spécifiques données par leur expression algébrique. Il est rare qu'ils aient eu l'occasion de manipuler une famille de fonctions dépendant de paramètres et de travailler avec des fonctions sous contraintes [12]. Les exercices sur les suites et les fonctions sont proposés à l'université avec une question ou deux, alors que dans le lycée des exercices analogues contiennent 5 questions et plus.

A l'université, les démonstrations occupent une place importante dans les cours d'Analyse et fournissent des méthodes de travail alors qu'au lycée, on s'intéresse aux résultats et ses applications.

\subsection{En Algèbre}

Pour l'algèbre, les difficultés des nouveaux étudiants sont dues au caractère formel, générateur et unificateur de l'algèbre linéaire. Pour Dorier et al "l'algèbre linéaire n'est qu'un catalogue de notions très abstraites qu'ils n'arrivent pas à se représenter; de plus ils sont submergés sous une avalanche de mots nouveaux, de symboles nouveaux, de définitions nouvelles et de théorèmes nouveaux. \ [8]. Les enseignants interviewés affirment que les étudiants ont des difficultés à manipuler des objets plus complexes que ceux du secondaire et vidés de sens et que ces difficultés sont interprétées comme résultant de « l'obstacle de formalisme » de l'algèbre linéaire.

Sierpinska ajoute que :

«L'obstacle du formalisme se manifeste chez les étudiants qui opèrent sur la forme des expressions, sans considérer ces expressions comme faisant référence à autre chose qu'à elles-mêmes. Un des symptômes en est la confusion entre différentes catégories d'objets mathématiques; par exemple, les ensembles sont traités comme des éléments d'ensembles, les transformations comme des vecteurs, les relations comme des équations, les vecteurs comme des nombres, et ainsi de suite. L'obstacle du formalisme fait produire aux étudiants un discours qui a les apparences du discours utilisé par l'enseignant ou le manuel. Pour être efficaces en tant qu'étudiants, ceux-ci vont souvent développer des automatismes. Un de ces 
automatismes est de construire une matrice et de réduire à chaque fois qu'ils le peuvent, quelle que soit la question qui leur est demandée. » [13].

L'entrée dans le monde des structures algébriques (Espaces vectoriels, applications linéaires) représente pour les élèves entrant à l'université un changement culturel [8]. L'algèbre linéaire fait appel à une multiplicité de langages et de registres de représentations (géométrique, algébrique et abstrait [14,15]. L'articulation des langages et des registres de représentations constitue une difficulté pour les nouveaux étudiants, le degré d'utilisation des règles de la logique est très élevé dans l'algèbre à l'université.

\subsection{En Géométrie}

Chaque cycle d'enseignement a sa spécificité dans la façon d'apprendre la géométrie [16].

- Au collège, on enseigne la géométrie comme « la première science physique », on étudie les figures planes, les solides de l'espace et les formules trigonométriques ;

- Au lycée, on commence à ajouter des notions telles que vecteurs, barycentre, produit scalaire, la géométrie analytique et la géométrie vectorielle ;

- A l'université, on introduit un modèle abstrait du plan ou de l'espace euclidien fondé sur l'algèbre linéaire.

Nous constatons un affaiblissement de la partie géométrique, surtout la géométrie plane.

\subsection{En Logique}

Les questions de logique et de langage se posent tout au long de la scolarité, mais elles sont plus cruciales au début des études universitaires à cause du formalisme. Les difficultés apparaissent surtout lors de la manipulation des énoncées comportant plusieurs quantificateurs $[17,18,19,20]$. Les étudiants de la première année rencontrent des difficultés dans l'emploi de l'implication, de la négation [21].

L'emploi des quantificateurs pose aussi problème aux étudiants surtout le quantificateur existentiel - \$ -. Vu l'importance des notions de logiques, il est nécessaire de les prendre en compte à la transition lycée-université.

Les enseignants interviewés affirment que les difficultés sont dues aux questions de la logique et de raisonnement auxquelles sont confrontés les nouveaux étudiants. Ces derniers sont démunis devant les questions de logique, ils ont des difficultés dans la production des preuves mathématiques [3]. Les enseignants du lycée sont sollicités à montrer l'utilité de la compréhension des rôles des quantificateurs et la maitrise de leur sens. Le secondaire prépare mal les élèves au formalisme et à la démonstration.

\section{Conclusion}

Dans cette étude, nous avons identifié les difficultés des étudiants en mathématiques lors de leur transition du lycée à l'université, nous avons identifié les causes de ces difficultés et nous avons dégagé les ruptures dans les pratiques et les modes d'enseignement entre les deux institutions, ces ruptures ont fait apparaître un écart entre l'enseignement secondaire et l'enseignement supérieur. La réduction de cet écart est à la charge des deux institutions

Des recherches et des perspectives de soutiens pédagogiques sont utiles. L'enseignement universitaire, par son formalisme est en opposition avec les approches adoptées au secondaire, cela est source de nombreux échecs [22] d'où la nécessité de prévoir au secondaire des situations qui travaillent la rationalité mathématique et vont donc être préparatoires au raisonnement dans des registres plus formels. 


\section{Recommandations}

Nous proposons les recommandations ci-dessous pour prendre en charge certaines difficultés repérées lors de l'enseignement-apprentissage des mathématiques, aider les élèves à préparer leur passage vers l'enseignement supérieur et diminuer le taux d'échec en première année d'université :

- Faciliter sur le plan institutionnel, des rencontres autour de la question de la « Transition lycée-université »;

- Créer des groupes de travail sur des thèmes de la transition ;

- Concevoir des dispositifs de soutien pédagogique pour les étudiants en difficultés comme le cas en France : - Math-Bridge : une stratégie remédiation individualisée ; - CapLicence: dispositif d'aide à la transition lycée-université.

Les deux projets ont le même objectif qui est l'amélioration de la réussite en première année universitaire. Pour CapLicence, La remédiation est effectuée avant la rentrée, alors que Math-Bridge s'étend sur toute la première année.

- Repenser l'orientation au lycée ;

- Aider les étudiants de la première année universitaire à passer de la culture secondaire à la culture universitaire;

- Réviser les programmes pour assurer une continuité entre le lycée et l'université.

- Lutter contre les mauvaises pratiques au lycée : - Donner des cours (parfois le résumé et les formules seulement) sans démonstrations - Examens nationaux de mathématiques contiennent le même type d'exercices.

- Implication des didacticiens, des inspecteurs et des enseignants des deux institutions dans la rédaction des manuels scolaires du secondaire ;

- Ne pas reprendre à l'université des parties de mathématiques enseignées au lycée surtout en cours magistraux.

\section{Références}

1. M. Artigue, Le défi de la transition secondaire supérieur. Que peuvent nous apporter les recherches en didactique des mathématiques ? Récupéré du site Mathématiques de l'Académie de Toulouse : http:// pedagogie.ac-toulouse.fr/math (2007)

2. M. Artigue, La didactique des mathématiques face aux défis de l'enseignement des mathématiques. In Gueudet, G. \& Matheron, Y. (dir.) Actes du séminaire national de didactique 2007, 14-45 (IREM de Paris7, 2008).

3. V.Durand-Guerrier, G.Arsac, Méthodes de raisonnement et leurs modélisations logiques. Spécificité de l'analyse. Quelles implications didactiques ? Recherches en Didactique des Mathématiques. 23(3), 295-342 (2003).

4. G.Gueudet, Entrée à l'université / Ressources en ligne. Éclairages théoriques et actions didactiques dans deux champs de recherche en didactique des mathématiques (note de synthèse pour l'habilitation à diriger des recherches,). (Université Paris 7, 2008). Récupéré de l'archive HAL : http://hal.archives-ouvertes.fr

5. Robert, A, Outil d'analyse des contenus mathématiques à enseigner au lycée et à l'université. Recherches en Didactique des Mathématiques.18 (2), 39-190(1998).

6. D. Grenier, C. Payan, 'Situation de recherche en classe : essai de caractérisation et proposition de modélisation', Cahiers du séminaire national de recherche en didactique des mathématiques, (Paris, 2003).

7. I. Bloch, I. Ghedamsi, Comment le cursus secondaire prépare-t-il les élevés aux études universitaires ? Le cas de l'enseignement de l'analyse en Tunisie. Petit x, 69, 7-30 (2005). 
8. J.-L.Dorier, A.Robert, J. Robinet, M. Rogalski, L'algèbre linéaire: l'obstacle du formalisme à travers diverses recherches de 1987 à 1995. In Dorier, J.-L (dir.). L'enseignement de l'algèbre linéaire en question, (105-147). (Grenoble : La Pensée Sauvage, 1997).

9. M.-P. Chopin, Le temps didactique et ses niveaux d'étude : enjeux d'une clarification conceptuelle pour l'analyse des pratiques d'enseignement. Recherches en didactique des mathématiques. 30(1), 83-112(2010).

10. I. Bloch, P.Gibel, A model to analyze the complexity of calculus knowledge at the beginning of university course Presentation and examples. Annales de didactique et de sciences cognitives, 24, 183 - 206(2019).

11. S. Coppe, J.-L.Dorier, I.Yavuz De l'usage des tableaux de valeurs et des tableaux de variations dans l'enseignement de la notion de fonction en France en seconde. Recherche en Didactique des Mathématiques. 27 (2),151-186(2007).

12. I. Bloch, L'enseignement de l'analyse à la charnière lycée / université. Savoirs, connaissances et conditions relatives à la validation. Thèse de doctorat. (Université de Bordeaux 1, 2000).

13. A. Sierpinska, T. Dreyfus, J. Hillel, Evaluation of a Teaching Design in Linear Algebra : the Case of Linear Transformations. Recherches en didactiques des mathématiques. 19(1), 7-40 (1999).

14. J. Hillel, Mode of description and the problem of representation in linear algebra. In J.L. Dorier (ed.). On the teaching of linear algebra.191-207. (Drdrecht : Kluwer Academic Publishers, 2000).

15. A. Sierpinska, One some aspects of students' thinking in linear algebra. In J.L. Dorier (ed.). On the teaching of linear algebra.209-246. (Drdrecht : Kluwer Academic Publishers, 2000).

16. C. Houdement, A. Kuzniak, Formation des maîtres et paradigmes géométriques, Recherche en Didactique des Mathématiques. 20(1), 89-116(2000).

17. J. Selden, \& A. Selden, Unpacking the logic of mathematical statements. Educational Studies in Mathematics, 29,123-151(1995).

18. E. Dubinsky, O. Yiparaki, On students understanding of AE and EA quantification. Research in Collegiate Mathematics Education IV, CBMS Issues in Mathematics Education, 8, 2000, 239-289. (American Mathematical Society : Providence, 2000).

19. J. Rogalski, M. Rogalski, Contribution à l'étude des modes de traitement de la validité de l'implication par de futurs enseignants de mathématiques. Annales de Didactique et de Sciences Cognitives, 9, Actes du colloque Argentoratum de juillet 2002, 175-203. (2004).

20. F. Chellougui, L'utilisation des quantificateurs universels et existentiels en première année universitaire entre l'explicite et l'implicite. Thèse des universités Lyon 1 et Tunis. (2004).

21. I. Ben Kilani, Effets didactiques des différences de fonctionnement de la négation dans la langue arabe, la langue française et le langage mathématique. Thèse en co-tutelle des Universités Lyon 1 et Tunis. (2005).

J. P. Drouhard, Prolégomènes " épistémographiques " à l'étude des transitions dans l'enseignement des mathématiques. Actes du colloque EMF 2006 : L'enseignement des mathématiques face aux défis de l'école et des communautés, (Sherbrooke (Québec) : Canada, 2006) - http://halshs.archives-ouvertes.fr/halshs-00426602. 